\title{
Transporte de Contaminantes en Aguas Subterráneas mediante Redes Neuronales Artificiales
}

\author{
Ignacio García ${ }^{(1)}$, José G. Rodríguez ${ }^{(2)}$, Felipe López $^{(2)}$ y Yenisse M. Tenorio ${ }^{(2)}$ \\ (1) Instituto Politécnico Nacional, Centro Mexicano para la Producción Más Limpia, \\ Depto. de Posgrado, Av. Acueducto s/n, Barrio La Laguna, Col. Ticomán, Del. Gustavo A. Madero, \\ 07340 México, D. F.-México (e-mail: igarcias@ipn.mx) \\ (2) Instituto Politécnico Nacional, Escuela Superior de Ingeniería y Arquitectura, Sección de Estudios \\ de Posgrado e Investigación, U. Prof. "Adolfo López Mateos", Zacatenco, Del. Gustavo A. Madero, \\ 07738 México, D. F.-México (e-mail: adore81@prodigy.net.mx; ytenoriom0700@ipn.mx; \\ flopezs@ipn.mx)
}

Recibido Oct. 23, 2009; Aceptado Nov. 25, 2009; Versión Final recibida Ene. 21, 2010

\begin{abstract}
Resumen
Se aplicó un modelo de Red Neuronal Artificial para predecir el transporte de contaminantes (cobre y cadmio) en medios saturados, homogéneos e isotrópicos para diferentes clases texturales. Los modelos fueron entrenados y evaluados a partir de la ecuación propuesta por Ogata y Banks, que considera los términos advectivo y difusivo. Se desarrollaron estructuras de retropropagación empleando una estructura de tres capas, considerando 4, 7 y 10 neuronas en la capa oculta. Para el entrenamiento y simulación se empleó el algoritmo de Levenberg-Marquardt; se aplicó la función de transferencia Log-sigmoidal en la capa oculta, y lineal en la capa de salida. La elección de los modelos se efectuó en base al coeficiente de correlación y al mínimo error de generalización. Se comprobó que la Red Neuronal Artificial es una herramienta matemática útil, de bajos requerimientos computacionales y con la suficiente eficiencia para estimar el transporte de contaminantes en medios porosos saturados, homogéneos e isotrópicos.
\end{abstract}

Palabras clave: redes neuronales artificiales, retropropagación, transporte de contaminantes, clases texturales

\begin{abstract}
An Artificial Neural Network model was developed for predicting pollutants transport (cupper and cadmium) in saturated, homogeneous and isotropic media for several textural classes. The models were trained and evaluated from the equation proposed by Ogata and Banks that considers advective and diffusive terms. Backpropagation structures were developed, using a three-layer architecture considering 4, 7 and 10 neurons in the hidden layer. For training and simulation the LevenbergMarquardt algorithm was used, the Log-sigmoid transfer function was applied in the hidden layer and a linear function was applied in the output layer. The results demonstrate that the Artificial Neural Network is a useful mathematical tool; it has low computational requirements and allows estimating the transport of pollutants in saturated, homogeneous and isotropic media.
\end{abstract}

Keywords: artificial neural networks, backpropagation, pollutants transport, textural classes 


\section{INTRODUCCIÓN}

A nivel mundial existen problemas de contaminación de aguas subterráneas generados por el uso de plaguicidas, derrames de combustibles y actividades mineras. Debido a esto, se plantea el uso de las Redes Neuronales Artificiales (RNA) como un método alternativo para predecir el transporte de contaminantes, respecto a métodos existentes, como son las Diferencias Finitas y Elemento Finito, mismos que exigen bastante información y son complejos en su calibración. (Muhammad, 2004). En fechas recientes se han desarrollado nuevos métodos numéricos, los cuales presentan una mayor flexibilidad, eficacia y precisión. Un ejemplo son las RNA, las cuales son una herramienta computacional de aprendizaje adaptativo, con capacidad de generalización y de fácil inserción dentro de la tecnología existente. Por sus características, las RNA generalmente presentan bajos requerimientos computacionales y su construcción es menos compleja. (Muhammad, 2004)

El uso de esta herramienta se ha incrementado en los últimos años principalmente en el área ambiental realizando el pronóstico de concentraciones de $\mathrm{O}_{3}, \mathrm{PM}_{10}$ y $\mathrm{PM}_{2.5}, \mathrm{SO}_{2}, \mathrm{NO}_{3}$ (García et al., 2008; Perez y Reyes, 2006; Brunelli et al., 2006; Thomas y Jacko, 2007; Grivas y Chaloulakou, 2005); evaluando la calidad del agua subterránea considerando diversos parámetros fisicoquímicos (Kuo et al., 2003); y prediciendo la distribución de Nitrato en aguas subterráneas (Wang et al., 2006; Yesilnacar et al., 2007); así como en Hidráulica, prediciendo porosidades, permeabilidades, conductividades hidráulicas en suelos (Helle, et al., 2001; García y Shigidi, 2005), y fluctuaciones del nivel superficial de lagos. (Altunkaynak, 2006)

En este trabajo se muestra un modelo de RNA del tipo de retropropagación en multicapas para cada clase textural de las doce consideradas por el Departamento de Agricultura de los Estados Unidos, que predice la movilidad de elementos disueltos no reactivos en materiales saturados, homogéneos e isotrópicos por efecto de los fenómenos de advección, dispersión mecánica y difusión molecular en una dimensión, entrenados y evaluados con la ecuación de advección - dispersión propuesta por Ogata y Banks (1961).

\section{MATERIALES Y METODOS}

La RNA fue aplicada a cada clase textural, considerando para cada una la porosidad efectiva $\left(\theta_{\mathrm{e}}\right)$, y la conductividad hidráulica $(\mathrm{K})$ en $\mathrm{cm} / \mathrm{h}$ (Tabla 1). En estos materiales se estudio el transporte de los cationes cobre y cadmio, con coeficientes de difusión $\left(D_{d}\right)$ de 7.33 y $7.17 \mathrm{~cm}^{2} / \mathrm{s}$, respectivamente.

Tabla 1: Clasificación de las clases texturales del suelo de acuerdo con el Departamento de Agricultura de los Estados Unidos; así como las porosidades efectivas y conductividades hidráulicas de las 12 clases texturales. (Charbeneau, 2000 y Bedient et al., 1994)

\begin{tabular}{|l|c|c|}
\hline \multicolumn{1}{|c|}{ Clases Texturales } & $\begin{array}{c}\text { Porosidad } \\
\text { efectiva }\left(\theta_{\mathrm{e}}\right)\end{array}$ & $\begin{array}{c}\text { Conductividad } \\
\text { Hidráulica }(\mathrm{K}) \\
(\mathrm{cm} / \mathrm{h})\end{array}$ \\
\hline A. Arcilla & 0.385 & 0.06 \\
\hline B. Arcilla limosa & 0.423 & 0.09 \\
\hline C. Arcilla arenosa & 0.321 & 0.12 \\
\hline D. Migajón arcillo limoso & 0.423 & 0.15 \\
\hline E. Migajón arcilloso & 0.309 & 0.23 \\
\hline F. Migajón arcillo arenoso & 0.330 & 0.43 \\
\hline G. Limo & 0.460 & 0.25 \\
\hline H. Migajón limoso & 0.486 & 0.68 \\
\hline I. Migajón & 0.434 & 1.32 \\
\hline J. Migajón arenoso & 0.412 & 2.59 \\
\hline K. Arena migajonosa & 0.401 & 6.11 \\
\hline L. Arena & 0.417 & 22.1 \\
\hline
\end{tabular}

La modelación se aplicó a un caso hipotético con el fin de evidenciar la aplicación de las RNA, empleando la ecuación general de transporte de contaminantes en medios porosos saturados, homogéneos e isotrópicos (ecuación 1), propuesta por Ogata y Banks (1961), que considera los 
fenómenos advectivos y difusivos en una dimensión, misma que ha servido como base para el desarrollo de los métodos tradicionales en este campo:

$$
\frac{C(x, t)}{C_{o}}=\frac{1}{2}\left[\operatorname{erfc}\left(\frac{x-v t}{2 \sqrt{D_{L}^{*} t}}\right)\right]
$$

Donde $C_{0}$ es la concentración inicial del contaminante, $C$ es la concentración del contaminante a una distancia y tiempo determinados ( $C / C_{0}$ es la concentración relativa), $x$ es la distancia de la trayectoria de flujo, $D_{L}$ es el coeficiente de dispersión hidrodinámico, calculado como:

$$
D_{L}=\alpha_{L} v+D^{*}
$$

que involucra el coeficiente de difusión molecular $\left(D^{*}\right)$ y el coeficiente de dispersitividad longitudinal:

$\left(\alpha_{L} \approx 0.0175 L^{1.46}\right)$

erfc es el complemento de la función error, $v$ es la velocidad lineal del agua subterránea, conocida como velocidad de Darcy:

$v_{X}=\frac{K \frac{h_{L}}{l}}{\theta_{e}}$

Ecuación válida para un flujo laminar, esto es, entre un número de Reynolds de entre 1 y 10.

La ecuación se resolvió en la Zona de Transición; esto es, desde donde se inicia el decaimiento de la concentración relativa del contaminante, conocida como atrás del frente advectivo $(x<<v t)$, en el que $C / C_{0}=1$; pasando por el frente advectivo $(x=v t)$, siendo $C / C_{0}=0.5$; hasta donde la concentración es cero, nombrado este punto, más allá del frente advectivo $(x>>v t)$, donde $C / C_{0}=0$ (Mora, 1999). Para la construcción de los modelos de RNA se generaron dos bases de datos a partir de la ecuación (1). La primera de ellas fue realizada con el objetivo de entrenar los modelos de RNA, y la segunda se empleó para la evaluación de dichos modelos.

Los modelos consideran como variables de entrada para cada clase textural la conductividad hidráulica $(K(\mathrm{~m} / \mathrm{s}))$; porosidad efectiva $\left(\theta_{e}\right)$; tiempo máximo de simulación de 1 año y 200 días $(t(s))$, para la fase de entrenamiento y evaluación, respectivamente. Se manejaron incrementos de distancia $(\Delta x(m))$, para calcular las concentraciones relativas; así como el coeficiente de difusión $\left(D^{*}\left(\mathrm{~m}^{2} / \mathrm{s}\right)\right)$. Para esta variable, en el entrenamiento se empleó el Cobre $\left(\mathrm{Cu}^{2+}\right)$ y para la fase de evaluación se simuló el Cadmio $\left(\mathrm{Cd}^{2+}\right)$, ya que a altas concentraciones son tóxicos para el ser humano (Volke y Velasco, 2005).

\section{RNA tipo de Retropropagación (Backpropagation)}

Se empleó el tipo de Red de Retropropagación en multicapas, qué por sus características ha sido empleada satisfactoriamente (García y Shigidi, 2005; Kuo et al., 2003; Helle et al., 2001; Yesilnacar et al., 2007; Yetilmezsoy y Demirel, 2007). La importancia de esta red consiste en su capacidad de autoadaptar los pesos de las neuronas de las capas intermedias para aprender la relación que existe entre un conjunto de patrones dados como ejemplo y sus salidas correspondientes. Para poder aplicar esa misma relación, después del entrenamiento, a nuevos vectores de entrada y dar salidas satisfactorias a entradas que el sistema nunca ha visto, característica conocida como la capacidad de generalizar de una RNA. (Mehrotra et al., 1997). Este tipo de redes consta de tres capas; la primera, una capa de entrada $A_{i}$ con $\boldsymbol{m}$ neuronas y una capa de salida $C_{k}$ con $\boldsymbol{o}$ neuronas y al menos una capa oculta $B_{j}$ con $\boldsymbol{n}$ neuronas internas. Cada neurona de una capa (excepto las de entrada) recibe entradas de todas las neuronas de la capa anterior y envía su salida a todas las neuronas de la capa posterior (excepto las de salida). 
Este algoritmo de aprendizaje involucra un paso de propagación hacia adelante, donde el patrón de entrada es presentado a la red y propagado a través de las capas hasta llegar a la capa de salida; seguido de un paso de propagación hacia atrás. En esta fase, los errores se transmiten hacia atrás, partiendo de la capa de salida, hacia todas las neuronas de la capa intermedia, hasta llegar a la capa de entrada, reajustando los pesos sinápticos, de manera que se logre la convergencia del sistema (Gurney, 2003), por lo que la red aprende a reconocer distintas características de los patrones de entrada (Freeman et al., 1993, Lek et al., 2000). La importancia radica en que cada iteración la RNA disminuye el error entre los datos reales y los valores pronóstico. Para el desarrollo de los modelo de RNA, se empleó el programa computacional MATLAB versión 7.4.0.287 (R2007a), en particular el módulo de herramientas "Neural Network Toolbox" (Wang et al., 2006, Yetilmezsoy y Demirel et al., 2008, García et al., 2008).

\section{Parámetros considerados para la construcción de la RNA}

El algoritmo de entrenamiento usado en los modelos de RNA fue Levenberg-Marquardt, debido a que ofrece una rápida convergencia (TRAINLM) (Demuth et al., 2005, Yetilmezsoy y Demirel et al., 2008 Yesilnacar et al., 2007, Wang et al., 2006), con una tasa de aprendizaje de 0.001; al aumentar este parámetro el desempeño de los modelos disminuía, y a valores menores el desempeño no se afectaba. Para evaluar los resultados de los modelos de RNA se aplicaron 2 funciones de desempeño: el error cuadrático medio (MSE) y la suma del error cuadrático (SSE). En la capa oculta, se empleó una función Log-sigmoidal (LOGSIG), y en la capa de salida, la función de transferencia fue de tipo lineal (PURELINE).

\section{Número de Capas ocultas y Neuronas en capa oculta}

Respecto al número de capas, en general los modelos de RNA tienen desempeños aceptables con tres capas: entrada, oculta y salida (Del Brío y Sanz, 2001, Yetilmezsoy y Demirel et al., 2008, Helle at al., 2001). En cuanto al número de neuronas en la capa oculta, la determinación de éstas no suele ser tan evidente, por lo se tomaron en cuenta las reglas sugeridas por Goethals et al., (2007), presentadas en la Tabla 2.

Tabla 2: Regla sugerida para el número de neuronas en la capa oculta basado en el número de variables de entrada (Ni) y/o nodos de salida (No). (Goethals et al., 2007)

\begin{tabular}{|c|c|}
\hline $2 / 3^{*} \mathrm{Ni}$ & $2 / 3^{*}(5)=3.33 \approx 3$ \\
\hline $0.75{ }^{*} \mathrm{Ni}$ & $0.75^{*}(5)=3.75 \approx 4$ \\
\hline $0.5^{*}(\mathrm{Ni}+\mathrm{No})$ & $0.5^{*}(5+1)=3$ \\
\hline $2{ }^{*} \mathrm{Ni}+1$ & $\left(2{ }^{*}(5)\right)+1=11$ \\
\hline $2{ }^{*} \mathrm{Ni}$ & $2{ }^{*}(5)=10$ \\
\hline
\end{tabular}

Con las presentes reglas, se determinaron los valores extremos del número de neuronas en la capa oculta de los 12 modelos de RNA para ser entrenados y evaluados, siendo arreglos de 4 y 10 neuronas. Se eligió un arreglo intermedio de 7 neuronas en la capa oculta, para tener una distribución cada 3 neuronas; con el objetivo de tener más opciones en la elección final de los modelos; se tomó esta decisión ya que no hay una regla que indique el número óptimo de neuronas en la capa oculta (Yetilmezsoy y Demirel et al., 2008, Helle et al., 2001). En cada problema se debe ensayar diferentes arreglos para organizar la representación interna y seleccionar la que proporcione mejores resultados de acuerdo a los objetivos planteados. (Rodriguez et al., 2006)

\section{RESULTADOS Y DISCUSION}

El desempeño de los modelos de RNA para cada clase textural fue evaluado con el coeficiente de correlación (r) (Yesilnacar et al., 2007) y el error mínimo de generación, conocido también como fenómeno de sobreentrenamiento. El coeficiente de correlación es la medida usual del grado de correlación basándose en una muestra de $n$ pares de observaciones. Si la correlación es fuerte, $r$ será cercano a 1 . Si $r=1$, significa que existe un ajuste perfecto. Cuando $r=0$, indica que no existe correlación. (Chao, 1998) 
En lo que respecta al fenómeno de sobreentrenamiento, al principio la red se adapta progresivamente al conjunto de aprendizaje, adaptándose al problema y mejorando la generalización, entendida como la capacidad de la red de dar una respuesta correcta ante patrones que no hayan sido empleados en su entrenamiento. Sin embargo, en un momento dado el sistema se ajusta demasiado a las particularidades de los patrones empleados en el entrenamiento, por lo que crece el error que comete ante diferentes patrones a los empleados en esta fase. En este momento la red no ajusta correctamente, sino que simplemente esta memorizando los patrones del conjunto de aprendizaje. Idealmente, dada una arquitectura de RNA, esta debería entrenarse hasta un punto óptimo en que el error de generalización, que es la diferencia entre el error de entrenamiento y evaluación, es mínimo. (Del Brío y Sanz, 2001). Para eliminar este fenómeno, durante el aprendizaje se deben representar a la vez la fase de entrenamiento y evaluación, para obtener el mínimo error de generalización. En la Tabla 3, se presentan los modelos de RNA para las 12 clases texturales, con el número óptimo de neuronas en la capa oculta y su respectiva función de desempeño, que tuvieron el mínimo error de generación y el coeficiente de correlación más cercano a 1.

Tabla. 3: Modelos de Redes Neuronales Artificiales seleccionados para las 12 clases texturales.

\begin{tabular}{|c|c|c|c|c|c|c|c|c|c|c|c|c|}
\hline Clase textural & A & B & C & D & E & F & G & H & I & J & K & L \\
\hline $\begin{array}{c}\text { Neuronas } \\
\text { capa oculta }\end{array}$ & 4 & 7 & 4 & 4 & 7 & 7 & 7 & 10 & 10 & 10 & 7 & 4 \\
\hline $\begin{array}{c}\text { Función de } \\
\text { Desempeño }\end{array}$ & MSE & MSE & SSE & MSE & SSE & MSE & MSE & SSE & MSE & MSE & MSE & MSE \\
\hline
\end{tabular}

Para la textura Migajón arcillo limoso, como ejemplo, tras una fase inicial en la que pueden aparecer oscilaciones en el valor del error, el error de aprendizaje tiende a disminuir, mientras que el error de evaluación a partir de la iteración 18 se incrementa, lo que indica una degradación progresiva del aprendizaje. En la Figura 1, la curva punteada representa la fase de entrenamiento, y la curva continua muestra el comportamiento del modelo en la fase de evaluación. Para la presente textura el mínimo error de generalización se presenta en la iteración 18, con un desempeño en la fase de entrenamiento de $2.945 \times 10^{-5}$ y en la fase de evaluación $2 \times 10^{-4}$, aproximadamente.

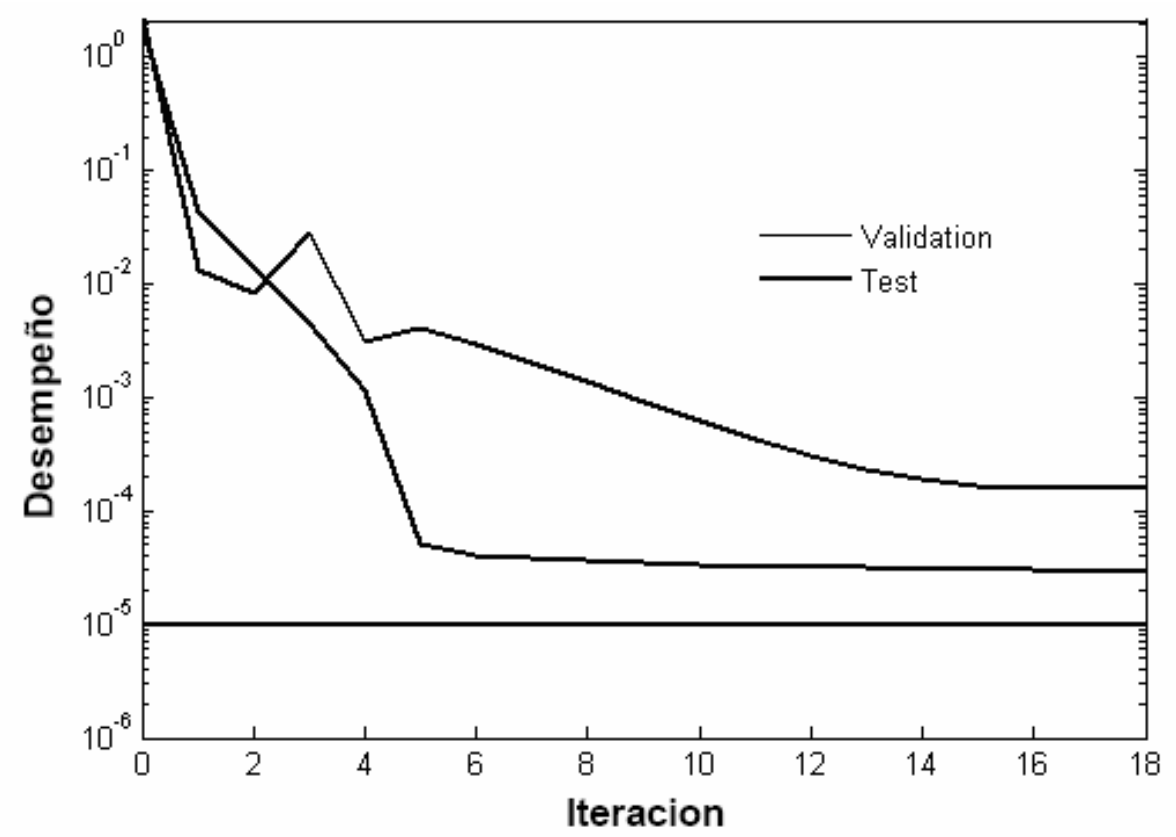

Fig. 1: Entrenamiento y evaluación de la RNA para la textura Migajón arcillo arenoso. 
En la figura 2(a) y 2(b), muestran la regresión lineal entre los valores proporcionados por la ecuación de Advección - Dispersión (A) y los valores de concentraciones relativas estimados por la Red Neuronal Artificial (T) en la fase de entrenamiento y evaluación, respectivamente.

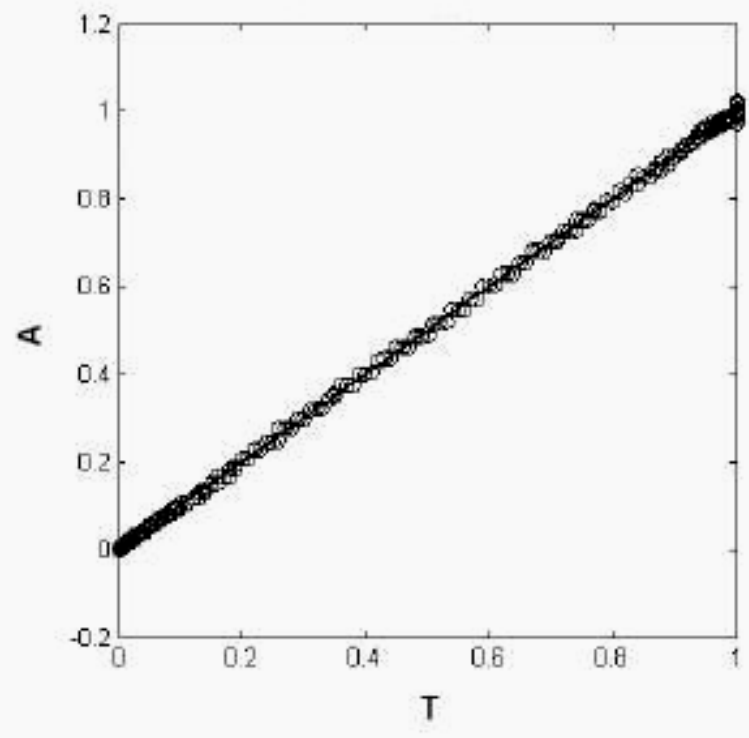

(a)

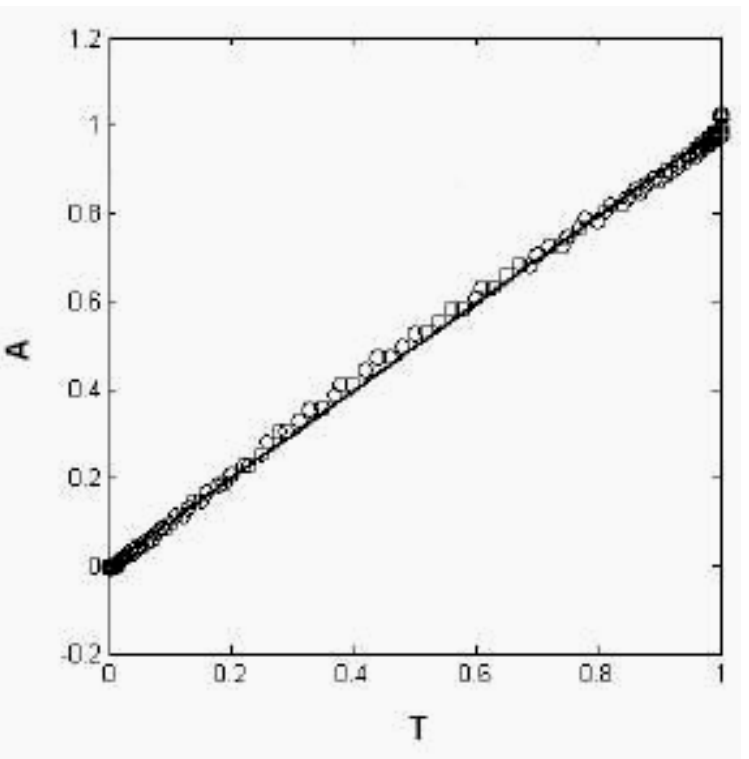

(b)

Fig. 2: (a) Correlaciones en la fase de entrenamiento. (b) Correlaciones en la fase de prueba para el Migajón arcillo arenoso.

En la Tabla 4, se muestra el Coeficiente de correlación ( $r$ ), de los 12 modelos en la fase de entrenamiento y evaluación, presentando valores de $r$ superior a 0.999 , por lo que el ajuste es casi perfecto $(r=1)$. Así mismo, se muestran los desempeños de ambas fases de los 12 modelos de RNA, y el número de iteración donde se encontró el mínimo error de generación.

Tabla 4: Desempeño y coeficiente de correlación ( $r$ ) en la etapa de entrenamiento y evaluación de los modelos de RNA's seleccionados para las 12 clases texturales.

\begin{tabular}{|c|c|c|c|c|c|}
\hline \multirow{2}{*}{$\begin{array}{c}\text { Clase } \\
\text { Textural }\end{array}$} & \multirow{2}{*}{ No. Iteración } & \multicolumn{2}{|c|}{$\begin{array}{c}\text { Etapa de } \\
\text { Entrenamiento }\end{array}$} & \multicolumn{2}{c|}{ Etapa de Evaluación } \\
\cline { 3 - 6 } & & Desempeño & $r$ & Desempeño & $r$ \\
\hline A & 800 & $6.340 \times 10^{-5}$ & 0.999 & $8.659 \times 10^{-4}$ & 0.999 \\
\hline B & 8000 & $2.455 \times 10^{-5}$ & 0.999 & $9.285 \times 10^{-3}$ & 0.988 \\
\hline C & 400 & 0.00585 & 0.999 & $4.359 \times 10^{-1}$ & 0.998 \\
\hline D & 1400 & $3.511 \times 10^{-5}$ & 0.999 & $1.340 \times 10^{-3}$ & 0.999 \\
\hline E & 60 & 0.0161 & 0.999 & $3.816 \times 10^{-2}$ & 0.999 \\
\hline F & 18 & $2.945 \times 10^{-5}$ & 0.999 & $1.835 \times 10^{-4}$ & 0.999 \\
\hline G & 3000 & 0.00732 & 0.999 & $5.085 \times 10^{-1}$ & 0.994 \\
\hline H & 170 & 0.00430 & 0.999 & $4.193 \times 10^{-2}$ & 0.999 \\
\hline I & 7 & $5.864 \times 10^{-5}$ & 0.999 & $1.074 \times 10^{-3}$ & 0.998 \\
\hline J & 1000 & $1.549 \times 10^{-5}$ & 0.999 & $4.371 \times 10^{-4}$ & 0.999 \\
\hline K & 150 & $1.603 \times 10^{-5}$ & 0.999 & $2.975 \times 10^{-4}$ & 0.999 \\
\hline L & 1200 & $3.378 \times 10^{-5}$ & 0.999 & $6.582 \times 10^{-4}$ & 0.999 \\
\hline
\end{tabular}




\section{CONCLUSIONES}

De acuerdo a los resultados de la Tabla 4, se muestra que los modelos no tienen una relación definida entre la textura y el número de neuronas en la capa oculta, lo que confirma que no hay una regla especifica que indique el número óptimo, por lo que en cada textura se debió ensayar con los distintos números de neuronas previamente seleccionados para organizar la representación interna y seleccionar la que presente mejores resultados.

No existe una relación entre la conductividad hidráulica y el número de neuronas ocultas, ya que estas últimas se eligen en base al desempeño que tienen los modelos de RNA en la fase de entrenamiento y evaluación. Aunque la conductividad hidráulica es importante en el transporte de contaminantes en medios porosos, los modelos cuentan con la porosidad efectiva y coeficiente de dispersión que intervienen directamente en el transporte de los contaminantes, por lo que no se puede establecer dicha relación entre la conductividad hidráulica y el número de neuronas en la capa oculta.

Los resultados de este artículo se obtuvieron con datos teóricos, ya que no se contaron con datos medidos en campo. En México, no se cuentan con referencias del uso de las RNA, por lo que este trabajo sirvió para evidenciar su aplicación en el área de transporte de contaminantes en aguas subterráneas, y de acuerdo a los resultados obtenidos se puede tomar en cuenta como una herramienta alternativa en esta área. Se comprobó que las RNA son una herramienta útil en el área ambiental, de bajos requerimientos computacionales y con la suficiente eficiencia para estimar el transporte de contaminantes en medios porosos saturados, homogéneos e isotrópicos.

\section{REFERENCIAS}

Altunkaynak, A.; Forecasting surface water level fluctuations of lake van by artificial neural networks, Water Resour Manage". 21, 399 - 408 (2006).

Bedient, P. B., H. S. Rifai y C. J. Newell; Ground water contamination: transport and remediation. Prentice Hall (1994)

Brunelli, U., y otros cuatro autores; Two-days ahead prediction of daily maximum concentrations of SO2, O3, PM10, NO2, CO I the urban area of Palermo, Italy, Atmospheric Environment". 41, 2967 2995 (2007).

Chao, L.L.; Introducción a la estadística; CECSA (1998).

Charbeneau R. J.; Groundwater Hydraulics and Pollutant transport. Prentice Hall (2000).

Demuth, H, M. Beale y M. Hagan; Neural Networks Toolbox. For Use with MATLAB. User's Guide Versión 4. The MathWortks (2005).

Del Brío, B. M. y A. Sanz; Redes Neuronales y Sistemas Borrosos, 2ª ed., Editorial Ra-Ma (2001).

Freeman, J. A. y Skapura D. M; Redes Neuronales: algoritmos, aplicaciones y técnica de programación. Díaz de Santos, 423 p. (1993).

García, I, A. Marbán, Y. M. Tenorio y J. G. Rodriguez; Pronóstico de la Concentración de Ozono en Guadalajara-México usando Redes Neuronales Artificiales, Información Tecnológica, 19(3), 89-96 (2008).

Garcia, L. A., A. Shigidi; Using neural networks for parameter estimation in ground water, Journal of Hydrology 318, 215-231(2006).

Goethals, P. L. M., y otros cuatro autores; Applications of artificial neural networks predicting macroinvertebrates in freshwaters, Aquat. Ecol., 41, 491 - 508 (2007). 
Grivas, G. y Chaloulakou A.; Artificial neural network models for prediction of PM 10 hourly concentrations, in the Greater Area of Athens, Greece, Atmospheric Environment". 40, 1216 - 1229 (2006).

Gurney, K.; An introduction to neural networks, CRC PRESS (2003).

Helle, H. B., Alpana B., Bjørn U.; Porosity and permeability prediction from wireline logs using artificial neural networks: a North Sea case study, Geophysical Prospecting”. 49, 431 - 444 (2001).

Kuo, Y., Chen-Wuing L., Kao-Hung L.; Evaluation of the ability of an artificial neural network model to assess the variation of groundwater quality in an area of blackfoot disease in Taiwan, Water Research". 38, 148 - 158 (2003).

Lek, S. y Guegan J. F; Artificial Neural Networks. Application to Ecology and Evolution, Springer, pp. $3-25(2000)$.

López, F.; Contaminación del Suelo por un Contaminante Conservativo, Tesis de Maestría, UNAM, D. F., México (1980).

Mehrotra, K., C. K. Mohar y S. Ranka; Elements of Artificial Neural Networks, The MIT Press Broadford (1997).

Mora, H. J; Modelo Matemático unidimensional de dispersión de contaminantes en aguas subterráneas, Tesis de maestría, IPN - ESIA TICOMAN, D. F., México (1999).

Ogata, A. y R. B. Banks; A Solution of the Differential Equation of longitudinal Dispersion in Porous Media. United States Government Printing Office. Paper 411 - A (1961).

Perez, P., Jorge R.; An integrated neural network model for PM10 forecasting, Atmospheric Environment". 40, 2845 - 2851 (2006).

Rodríguez, J. G. y M. Y, Tenorio; Desarrollo de Modelos Pronóstico para la calidad del Aire en la Zona Metropolitana de Guadalajara, Jalisco, Tesis de Licenciatura, ESIQIE-IPN, México (2006).

Shamim, M. A., Ghumman A. R., Ghani U.; TForecasting Groundwater Contamination using Artificial Neural Networks, "International Conf on Water Resources \& Arid Environment". (2004).

Thomas, S. y B. J. Robert; Model for forecasting expressway fine particulate matter and carbon monoxide concentration: Application of Regression and Neural Network Model, Air \& Waste Management Association". 57, 480 - 488 (2007).

Volke, T., J. A. Velasco y D. A. de la Rosa; Suelos contaminados por metales y metaloides: muestreo y alternativas para su remediación, Instituto Nacional de Ecología (2005).

Wang, M. X. Liu G. D., Wu W. L., Bao Y. H., Liu W. N.; Prediction of agriculture derived groundwater nitrate distribution in North China Plain with GIS-based BPNN, Environment Geology". 50, 637 - 644 (2006).

Yesilnacar, M. I., Erkan S., Naz M. y Bestamin O.; Neural network prediction of nitrate in groundwater of Harran Plain, Turkey, Environmental Geology". (2007).

Yetilmezsoy, K. y Demirel S.; Artificial neural networks (ANN) approach for modeling of Pb (II) adsorption from aqueous solution by Antep pistachio (Pistacia Vera L.) Shells, "Journal of Hazardous Materials". 153, 1288 - 1300 (2008). 